that, from a use perspective, the protective nature of salt has little clinical relevance. Although salt has been shown historically by many investigators to potentially impede hospital sterilization of medical devices, the emphasis of these results should be to highlight the need for thorough cleaning methodologies.

Acknowledgments. None.

Financial support. No financial support was provided relevant to this article.

Conflicts of interest. All authors report no conflicts of interest relevant to this article.

\section{References}

1. Rutala WA, Gergen MF, Sickbert-Bennett EE, Weber DJ. Comparative evaluation of the microbicidal activity of low-temperature sterilization technologies to steam sterilization. Infect Control Hosp Epidemiol 2020 [Epub ahead of print]. doi: 10.1017/ice.2020.2.

2. Guideline for disinfection and sterilization in healthcare facilities, 2008, updated May 2019. Centers for Disease Control and Prevention website. https://www.cdc.gov/infectioncontrol/pdf/guidelines/disinfection-guidelinesH.pdf. Published 2019. Accessed April 13, 2020.

3. Krebs MC, Becasse P, Verjat D, Darbord JC, Gas-plasma sterilization: relative efficacy of the hydrogen peroxide phase compared with that of the plasma phase. Int J Pharm 1998;160:75-81.

\title{
Reply to Randal W. Eveland regarding comparative evaluation of the microbicidal activity of low-temperature sterilization technologies to steam sterilization
}

\author{
William A. Rutala PhD, MPH${ }^{1}$, Maria F. Gergen MT(ASCP) ${ }^{3}$, Emily E. Sickbert-Bennett PhD, $\mathrm{MS}^{1,2}$ and \\ David J. Weber MD, MPH ${ }^{1,2}$ \\ ${ }^{1}$ Division of Infectious Diseases, University of North Carolina School of Medicine, Chapel Hill, North Carolina, ${ }^{2}$ Department of Hospital Epidemiology, University of \\ North Carolina Health Care, Chapel Hill, North Carolina and ${ }^{3}$ Formerly Department of Hospital Epidemiology, University of North Carolina Health Care, Chapel \\ Hill, North Carolina
}

To the Editor-We thank Dr Randal Eveland, Steris Corporation, for his letter regarding our paper that compared the microbicidal activity of low-temperature sterilization technologies (ie, vaporized hydrogen peroxide [VHP], ethylene oxide [ETO], and hydrogen peroxide gas plasma [HPGP]) to steam sterilization in the presence of salt and serum to simulate inadequate precleaning. ${ }^{1}$ As noted in our paper, the literature contains a paucity of information on the comparative microbicidal activity of the sterilization technologies cleared by the Food and Drug Administration (FDA) for sterilizing medical and surgical devices. We believe that the data from this study will help clinicians in infection prevention assess the robustness of healthcare sterilization technologies and the risk of infection to patients when an uncleaned instrument is unintentionally brought into the operating room or used on a patient.

We agree with Dr Eveland there are differences in concentration and duration of the VHP and HPGP cycles. Our experiments compared the microbicidal activity of FDA-cleared, low-temperature sterilization technologies to steam sterilization in the presence of salt and serum. The addition of salt and serum simulated inadequate cleaning of instruments prior to sterilization. We evaluated the "robustness" of sterilization technology that is used by hospitals throughout the United States. Robustness is defined as the ability to withstand and overcome adverse conditions or rigorous testing.

Concerning plasma in the HPGP technology, our intention was not to define the components of the cycle that created the robustness (eg, higher concentrations of hydrogen peroxide, plasma); it was

Author for correspondence: William A. Rutala, E-mail: brutala@med.unc.edu Cite this article: Rutala WA, et al. (2020). Reply to Randal W. Eveland regarding comparative evaluation of the microbicidal activity of low-temperature sterilization technologies to steam sterilization. Infection Control \& Hospital Epidemiology, 41: 1000-1001, https://doi.org/10.1017/ice.2020.239 solely to define whether FDA-cleared sterilization technologies had the same robustness or ability to inactivate microorganisms in the presence of organic matter and salt. Our results demonstrated that some sterilization technologies were more "forgiving" or safe when cleaning is not complete. Because protein (organic matter) remains on cleaned surgical instruments, ${ }^{2}$ we must investigate at what point the presence of protein overwhelms the ability of the sterilizer to inactivate contaminating microorganisms. Alternatively, we should consider using the most robust sterilization technologies that inactivate microorganisms in the presence of organic matter and salt when possible.

Regarding the comparison of HPGP to VHP and materials compatibility, there are other factors involved in materials compatibility than the hydrogen peroxide concentration alone. Although the theoretical concentration of hydrogen peroxide for HPGP is higher than for VHP (ie, 25.6 vs $9.1 \mathrm{mg} / \mathrm{L}$ hydrogen peroxide for the HPGP and VHP, respectively), the plasma process quickly removes the hydrogen peroxide from the load by dissociating unreacted hydrogen peroxide into oxygen and water, eliminating the need for aeration. ${ }^{3}$ The VHP sterilizer passes hydrogen peroxide through a catalytic converter where it is reduced to water and oxygen. The HPGP system has 3 potential advantages. First, because the plasma quickly removes the residual hydrogen peroxide, rather than a gradual release with VHP, there may be improved material compatibility and biocompatibility. However, we have not been able to find any data on the internet or in the peer-reviewed literature that demonstrated that VHP is more or less materials compatible or biocompatible than HPGP. Second, regarding environmental hydrogen peroxide levels, for both sterilizers there were no notable emissions from the sterilizers during the cycle. However, other investigators measured significant hydrogen peroxide emissions when the VHP chamber door was open compared 
to the HPGP (eg, hydrogen peroxide ranging from 5 to $17 \mathrm{ppm}$ for VHP vs $\leq 0.3 \mathrm{ppm}$ for HPGP). ${ }^{3}$ This demonstrated that HPGP emits less hydrogen peroxide into the breathing zone of the operator who opens the sterilizer door to remove the load. Third, the higher concentration of hydrogen peroxide in the HPGP sterilizer is a potential safety factor, which resulted in the significantly higher margin of safety for HPGP vs VHP (ie, failure rate of HPGP of $1.9 \%$ vs $76.3 \%$ for VHP).

As it pertains to FDA clearance and the test methodology, there are 2 issues. First, there are limitations to the effectiveness of sterilization technologies even though they are FDA-cleared and have been demonstrated to achieve a sterility assurance level of $10^{-6}$ under the test conditions. In our study, we assessed the margin of safety or robustness associated with the sterilization technologies currently used in healthcare facilities. Steam sterilization, which is most common and used for sterilization of instruments that are heat resistant, is the most effective and robust sterilization technology. Salt was the principal component that interfered with VHP, which is likely due to the salt crystals impeding the penetration of the sterilant to the microbe. Second, many salts (eg, most sodium, potassium and ammonium salts) are soluble in water and dissolve in water and are removed from surgical instruments when immersed in water ${ }^{4}$; however, some salts are insoluble or have a low solubility in water. Some salts, such as calcium carbonate, can occlude microbial exposure and dramatically affect the time required for inactivation. For example, the time required for inactivation of $8.0 \times 10^{3}$ Bacillus subtilis spores by ethylene oxide $(1,200 \mathrm{mg} / \mathrm{L})$ at $54^{\circ} \mathrm{C}$ with unoccluded spores is 30 seconds, but for spores occluded in calcium carbonate, the inactivation time is $>2$ weeks. ${ }^{5}$ Additionally, to understand the dynamics of a cleaning process and the potential effect of soils on a sterilization process, the different ingredients in the soil (ie, organic to inorganic ratio) need to be examined independently. ${ }^{4}$

Lastly, as demonstrated by this study, not all sterilization technologies used in healthcare to sterilize surgical instruments are equal and have the same robustness. Although FDA-cleared sterilization technologies theoretically kill a very large number of microorganisms on instruments (eg, 12 logs or a trillion), healthcare personnel can unintentionally impede the effectiveness of sterilization technology by improper cleaning of the instruments prior to sterilization. Cleaning, or the removal of visible soil and microbial contaminants from objects, precedes sterilization. If instruments are not properly cleaned prior to sterilization and are then placed in a low-temperature sterilization technology such as VHP, there is a possibility of failure. However, the robustness of some sterilization technology, such as steam sterilization, makes it exceedingly unlikely that a steam-sterilized instrument will be the source of infection. Most medical and surgical devices used in healthcare facilities are made of materials that are heat stable and thus are sterilized by heat, primarily steam sterilization. The data demonstrate how important cleaning is prior to sterilization because salt and organic matter left on instruments can interfere with low-temperature sterilization. These findings reinforce the need for meticulous cleaning and for reliable and validated cleaning monitoring methods that are predictive of microbial contamination and infection risk.

Acknowledgments. None.

Financial support. No financial support was provided relevant to this article.

Conflicts of interest. W.A.R. was a consultant to Advanced Sterilization Products (ASP) in 2019. All other authors report no conflicts of interest relevant to this article.

\section{References}

1. Rutala WA, Gergen MF, Sickbert-Bennett EE, Weber DJ. 2020. Comparative evaluation of the microbicidal activity of low-temperature sterilization technologies to steam sterilization. Infect Control Hosp Epidemiol doi: 10.1017/ ice.2020.2

2. Baxter RL, Baxter HC, Campbell GA, et al. Quantitative analysis of residual protein contamination on reprocessed surgical instruments. J Hosp Infect 2006;63:439-444.

3. Advanced Sterilization Products. Comparison study of environmental hydrogen peroxide levels of STERRAD systems and SRERIS V-PRO low temperature sterilizers reveals striking differences. chemDAQ website. https:// www.chemdaq.com/slides/embed/100?page=1. Accessed May 18, 2020.

4. Jacobs PJ, Wang J-H, Gorham RA, Roberts CG. Cleaning: Principles, methods and benefits. In: Rutala WA, editor. Disinfection, Sterilization and Antisepsis in Health Care. Champlain, NY: Polyscience Publications; 1998:165-181.

5. Doyle JE, Ernst RR. Resistance of Bacillus subtilis var. niger spore occluded in water-insoluble crystals to three sterilization agents. Appl Micro 1967; 15:726-730. 